\title{
Numerical Optimization applied to structure sizing at AIRBUS: A multi-step process
}

\author{
S. Grihon ${ }^{1 \mathrm{a}}$, L. Krog ${ }^{2}$, D. Bassir ${ }^{3}$ \\ ${ }^{1}$ AIRBUS, Toulouse, France, \\ ${ }^{2}$ AIRBUS, Filton, United Kingdom \\ ${ }^{3}$ TUDelft, Delft, The Netherlands.
}

Received 17 May 2009, Accepted 09 September 2009

\begin{abstract}
Structure optimization at airframe level is mainly focused on sizing design variables detailing the thin-walled properties of aircraft structures. Typical design variables are cross sectional dimensions for 1D and 2D elements with an additional complexity brought by composite materials with their directional and multi-layer aspects. Even if the scope of these design variables is clear and well understood, the vision of the structure behaviour is multi-criteria and encompasses various fidelity levels. Its design requires several stages from the future project to the detailed definition of structural parts using various analysis tools from different disciplines. These several stages require adequate structural optimization processes to offer the best response with the right level of details to answer questions being sought at each maturity level of the design. A review of methods \& tools developed and applied at AIRBUS to deliver automated sizing for aircraft structures along their development will be presented. Ways forward and major stakes for the future will be discussed.
\end{abstract}

Key words: structure optimization, aeronautical design, mathematical programming, composite materials, multi-level optimization

\section{Introduction}

In this paper a multi-step sizing process for aeronautical structures will be discussed. The described sizing process does not come from a single attempt at the problem, rather the process should be seen as a result of a multi-year effort in structural optimization [1-6]. For this reason it is important to first describe the history of structural optimization at AIRBUS, and show the development of ideas, which has lead to today's vision.

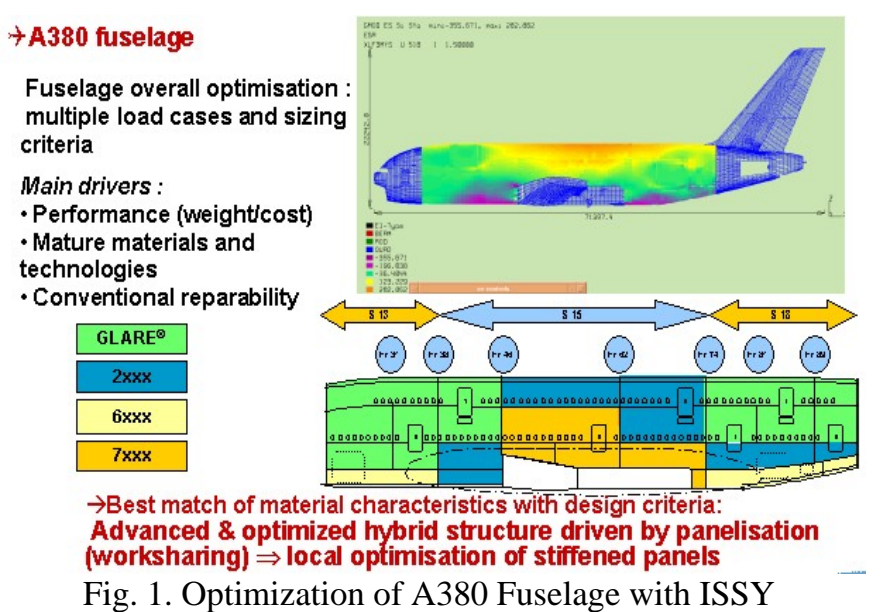

In a second part of the paper we describe sizing processes suited to support aircraft development from early concept design thought to detailed design. Three sizing stages have been identified and associated with sizing processes, which has been named: rapid sizing, preliminary sizing and detailed sizing. Sizing stages are described in reverse order of aircraft development starting from detailed sizing and going up to rapid sizing, integrating progressively the sizing process from the part level to the full aircraft level [7-10]. The two extremes of the sizing optimization process, which are considered today bottlenecks and therefore also the subject of intensive internal research, will then be discussed. This discussion considers how to address optimization at the finest level designing composite materials at ply level and considers how to address optimization at the highest level optimizing the full aircraft. Finally the consistency of the overall process and the continuity between the subsequent sizing stages will be commented. The conclusion will give the ways forward envisaged in the running projects

\section{A history of structural optimization at AIRBUS}

\subsection{From $A 340$ to $A 380$}

Structural optimization at AIRBUS was first implemented through sizing processes guided by engineer experience. Efficient tools, like ISSY (Fig. 1) for sizing of fuselages and later SOMBRA (Fig. 2) for sizing of wings, were developed implementing iterative re-sizing loops incorporating recalculation of internal loads. Today, tools are still used to provide an initial sizing for aircraft structures.

With the progress of computational means, sizing processes based on numerical optimization using gradient-based mathematical programming theory appeared. At the time where AIRBUS partners still had their own finite element software, in-house developments were made to add an optimization capability. An example of such early developments is ASELF from AIRBUS-France, which was developed to compute sensitivities of structural finite

(a) Corresponding author : stephane.grihon@airbus.com 
element responses and connected to gradient-based optimizers. Another example is the code STARS, which was developed in the 80s by ERA (today Qinetiq) in collaboration with AIRBUS-Germany. With the arrival of NASTRAN [11] as a common Finite Element (FE) package at AIRBUS, the optimization module of NASTRAN, SOL200, became widely used to address simple sizing optimization problems aiming to control direct NASTRAN responses. An example of a direct use of NASTRAN SOL200 is the work performed at the beginning of the A380 development to reduce running loads at the fuselage-wing intersection (Fig. 3).

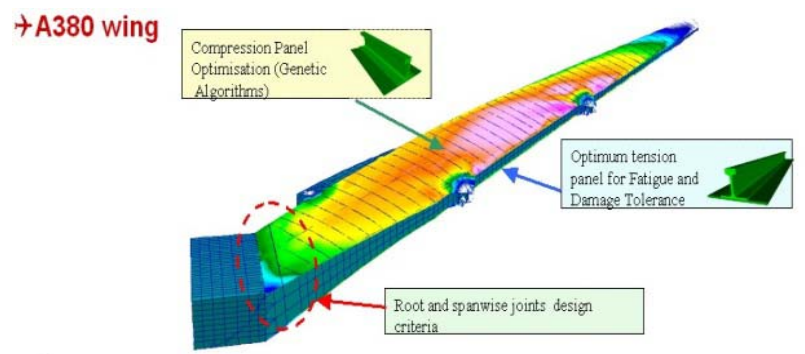

Fig. 2. SOMBRA Optimization of an A380 Wing (The SOMBRA sizing optimization is performed to determine optimum skin/stringer cross sections for a range of loading. Optimised local designs are stored into design curves that in the second level are used to update finite element properties in an iterative and global re-sizing process).

The use of SOL200 is here limited to stress allowable and running loads constraints (direct NASTRAN responses). NASTRAN was also used to compute sensitivities of global responses and chain these in complex multidisciplinary optimization processes. An example is the flutter and stress optimization chain developed in the 90s by AIRBUS-France. This was a stiffness driven sizing process and a first Multidisciplinary Design Optimization experience at AIRBUS.

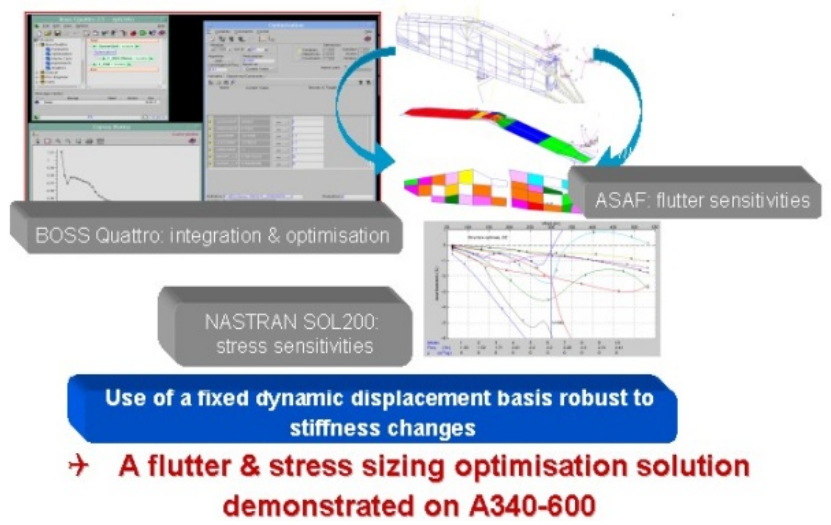

Fig. 3. Integrated flutter and stress optimization applied to A340-600 engine pylons

Another and more advanced example on a direct use of NASTRAN SOL200 is the SOL tool developed for sizing of composite wings (Fig. 3). The SOL tool embeds not only strain requirements but also engineer equations for buckling into a NASTRAN SOL200 input deck to provide a fast preliminary sizing and laminate optimization capability for a composite wing box.

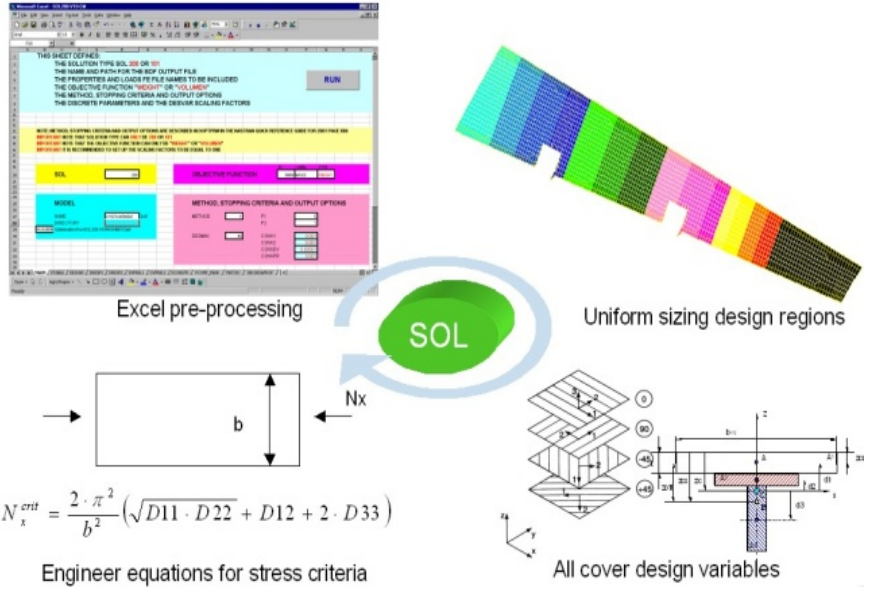

Fig. 4. Fast preliminary sizing of wing boxes with SOL

(SOL200 customization)

At the same time a parallel trend was to investigate sizing optimization based on more complex stress tools. These tools are the stress tools used for hand sizing at AIRBUS. They are generally still based on analytical (semi-empirical) approaches, but they chain a long list of equations with potential branching conditions (if then else) and/or integrate iterations and solvers (eigenvalue calculations for example). Such skill tools cannot be easily integrated in SOL200 as done for engineer equations in SOL.

However, due to the restricted number of variables and the small computation times, it was easy by using finite differences to integrate these skill tools inside open optimization frameworks and build automated sizing tools. A good example of application for this kind of tool is the weight saving campaigns made for A380, where many stiffened panels have been optimized thanks to fast integration of stress tools inside an open framework for optimization such BOSS Quattro [12-15] software (Fig. 5).

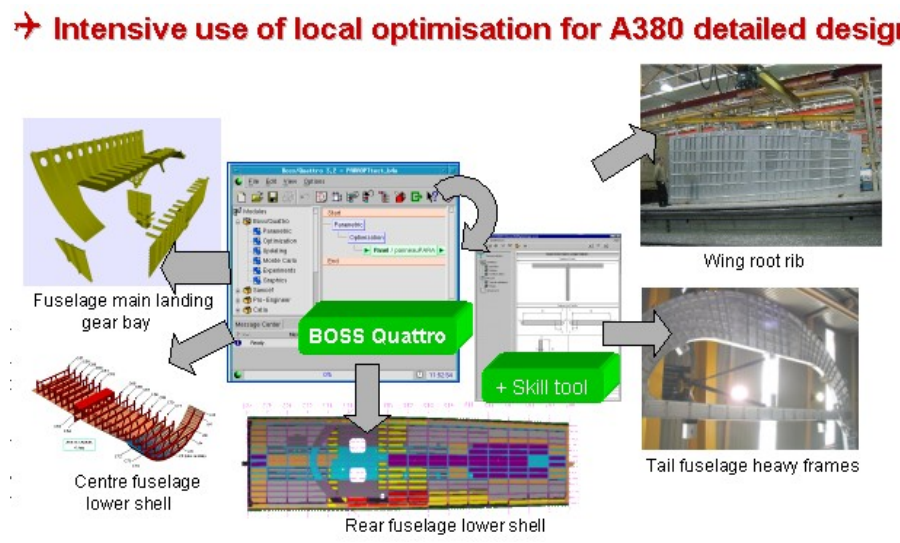

Fig. 5. A380 Weight Savings

\subsection{For A350 XWB}

It is important at this stage to make understand the sizing process of a structure at AIRBUS to position the various approaches.

Typically tools like SOMBRA, ISSY would automate this sizing process, but without considering more sophisticated stiffness effects, like control of internal load paths. In a way it is just an automation of a set of local optimizations with internal loads updates. Also processes, automating a set of 
local optimizations, would have difficulties dealing with global continuity aspects for composite designs.

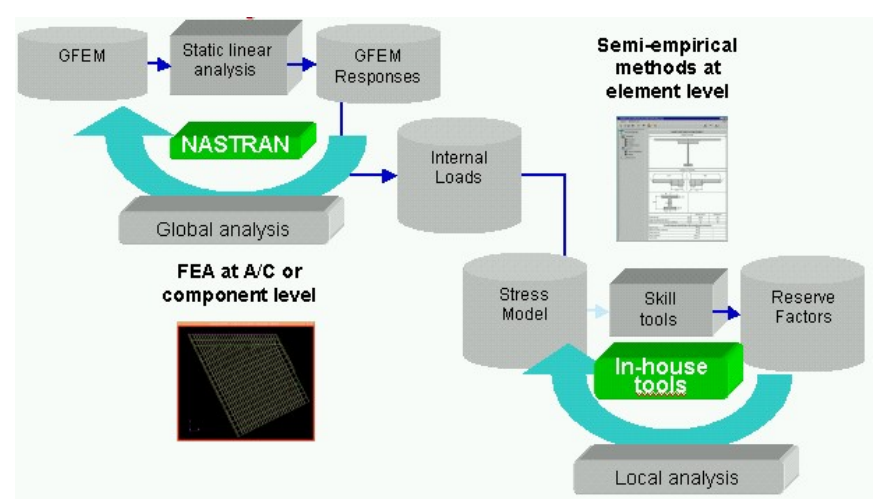

Fig. 6. Hand stress process at AIRBUS

So regarding the sizing approaches based on optimization or mathematical programming it is interesting to make a difference between optimization tools before and after A350. Before A350 there were essentially two kinds of optimization: global optimizations and local optimization. A good example of global optimization is the SOL process. Here the added value is in the consideration of internal load sensitivities with the potential to drive an optimum load path. It is also possible to add constraints on elements not considered in the optimization process with some elements not necessarily driven by stress considerations. This kind of approach is interesting for conceptual design but as the exact stress tools are not used but simplified engineering equations it is not applicable to further stages and especially to the preliminary sizing where a consolidated sizing is required. Another approach is local optimization. Local optimization means essentially that the Global Finite Element Model is not inside the optimization process. A good example is the use of local optimization for A380 weight saving campaigns. This kind of optimization is often made based on fixed internal loads applied to the part to be sized. For this reason it is limited to detailed design (Fig.7). Finally and more recently, a synthesis was made to integrate the first family of tools based on FE analysis and the second level based on stress tools. A tool was developed to mix FE based optimization with skill tool based.

\subsection{Summary}

Across this history, it is quite clear that there exist 3 families of tools:

- tools based on simple stress criteria and focused on GFEM behavior and stiffness criteria (aeroelastics, loads). These tools are to be used in early design stages in cooperation with Flight Physics to have a good initial sizing of the airframe. An exact stress analysis is not necessary here.

- tools based on advanced stress criteria and using GFEM to address the redistribution of internal loads or still considering some stiffness constraints. These tools have to be used in the transition from architecture to detailed design to consolidate the sizing before finer studies. This is the kind of tool to be used for preliminary sizing to have a good vision of the overall component sizing.

- tools based on skill tools again and focused on the use of skill tools as stand alone. This is the kind of tool to be used for detailed sizing to address part by part sizing. In certain cases we can also have fully FE based optimization.

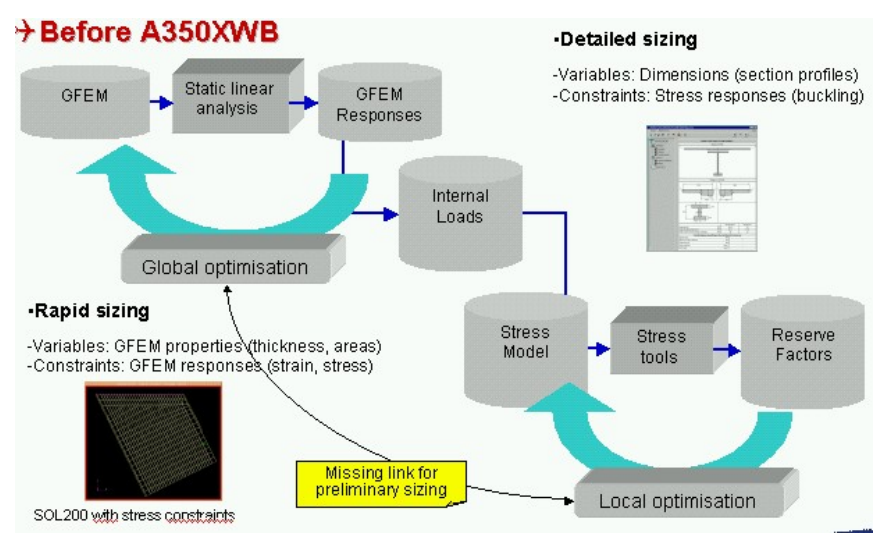

Fig. 7. Sizing optimization approaches before A350XWB

\section{A multi-step design process requires various sizing solutions}

It is standard in the aeronautical community to see the aircraft design split along three subsequent stages:

- conceptual design

- preliminary design

- detailed design

Conceptual design is an upstream task to optimize the aircraft configuration in terms of high level parameters like external geometry (3-side view), structural lay-out (frame and rib pitch), big choices in terms of materials (composite or metallic). Preliminary design is addressing more refined trade-offs selecting stringer pitch, choosing ply angles and addressing best compromises in terms of weights and costs. Detailed design comes after a good overall definition of airframe external structure (covers) and will deal with the detailed design of all structural parts down to system brackets for example. A way at AIRBUS to distinguish these three different stages is to define milestones linked to the development of an aircraft.

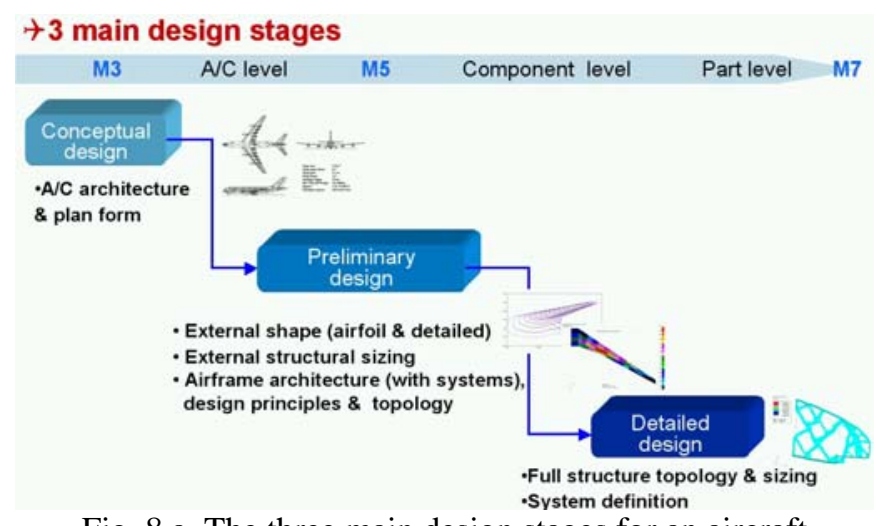

Fig. 8.a. The three main design stages for an aircraft

The main milestones for optimization are:

- M3: the aircraft concept is selected and the optimization of the configuration is starting at aircraft level 
- M5: The detailed aircraft configuration is validated and the detailed definition is starting

- M7: The definition is completed at component level and the manufacturing is starting

- M5 is an important milestone because from M5, the structure definition will be organized into work packages and addressed to various partners across the world (work sharing).

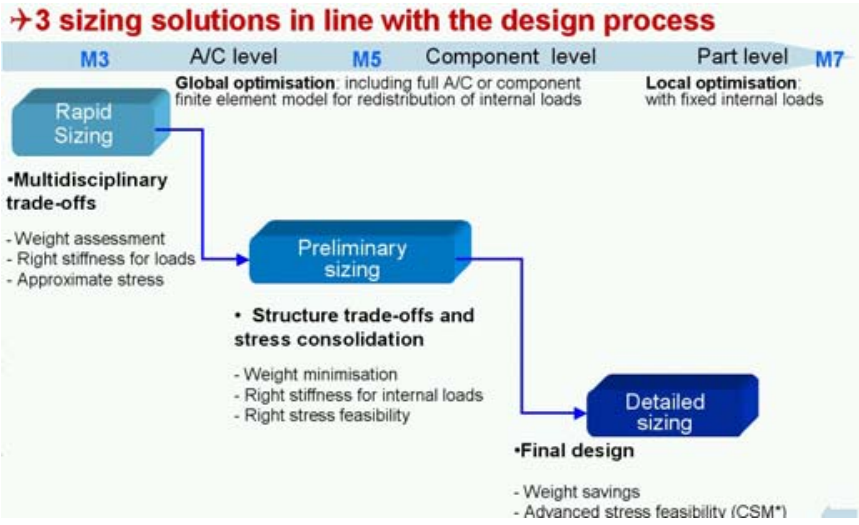

Fig. 8.b. The three sizing solutions proposed to support the three main design stages

For each stage and depending on the level of trade-offs and on the maturity of data, it is necessary to use a particular sizing process and particular sizing methods \& tools (Fig. 8 a $\& b$ ). For multidisciplinary trade-offs, when the focus is on weight assessment, we can accept an approximate stress estimate and use simplified equations. The number of open design variables can also be reduced through assumptions for secondary ones. For structure trade-offs and stress consolidation required before sharing the structure design work, it is necessary to consider agreed stress criteria and proper weight minimisation to give the right stiffness for external and internal loads. The final design focuses on latest weight savings and can used Computational Structure Mechanics for final design checks and updates. We will further describe each design stage detailing the way it was implemented today at AIRBUS. This description will be made from the detailed sizing addressing the most refined level of details up to the full aircraft level and upstream design stages.

\section{Detailed sizing}

Detailed sizing is the lower level of sizing. There are different approaches to address this detailed sizing. The problem to be solved is almost always the same and can be formulated as:

$$
\begin{gathered}
\operatorname{Min} \quad w(x) \\
x \in \Re^{n} \\
\text { s.t. }\left\{\begin{array}{l}
R F_{j}\left(x, N_{k}\right) \geq 1 \quad \mathrm{j}=1, \mathrm{n}_{\mathrm{c}} ; \mathrm{k}=1, \mathrm{n}_{\mathrm{lc}} \\
d_{l}(x) \geq 0 \quad \mathrm{l}=1, \mathrm{n}_{\mathrm{d}}
\end{array}\right.
\end{gathered}
$$

The loading is kept constant, there are only local design constraints and reserve factor constraints for stress feasibility $(R F>1)$

Note that internal loads are here to be considered as external loads because we are at part level. So this optimization, especially if the analysis is finite element based can include an internal load re-distribution (for the part). These different approaches depend on the way the part to be optimised is analysed and justified by stress people. Typically if we consider primary structures and especially external structures (covers) there are very particular design criteria like reparability and damage tolerance (especially for composite structures), which suppose to use analytical skill tools based on semi-empirical approaches. Other structures are less linked to such certification criteria and can be directly addressed via pure numerical approaches like refined finite element analysis. These major trends for the stress calculations orient to different approaches.

In the first case, sensitivities are often calculated via finite differences and it is practical to integrate the process inside an open optimization framework to perform the optimization. This is the approach used in the A380 weight saving campaign and pursued today on the A350XWB. This kind of approach is particularly in line with the works of stress people in the detailed design phase, because they often need to adapt the stress process and stress calculations and have to be very reactive in terms of sizing delivery. The possibility with BOSS Quattro for example to quickly assemble a calculation process, integrate and encapsulate inside an optimization process based on finite difference sensitivities is particularly suited to this care of reactivity. In the second case, the optimization process is more numerically based and requires advanced sensitivity analysis based on semianalytical approaches. This is particularly the case when addressing buckling or post-buckling. For this kind of optimization various software can be used linked to the kind of optimization performed. For pure sizing optimization, the use of NASTRAN SOL200 is frequent based on detailed model dedicated to buckling analysis.

When topology or shape variables are considered [16-17] it is often more efficient to use ALTAIR Hyperworks suite for its pre-processing capabilities (Fig. 9), but the process is about the same: FE-based optimization.

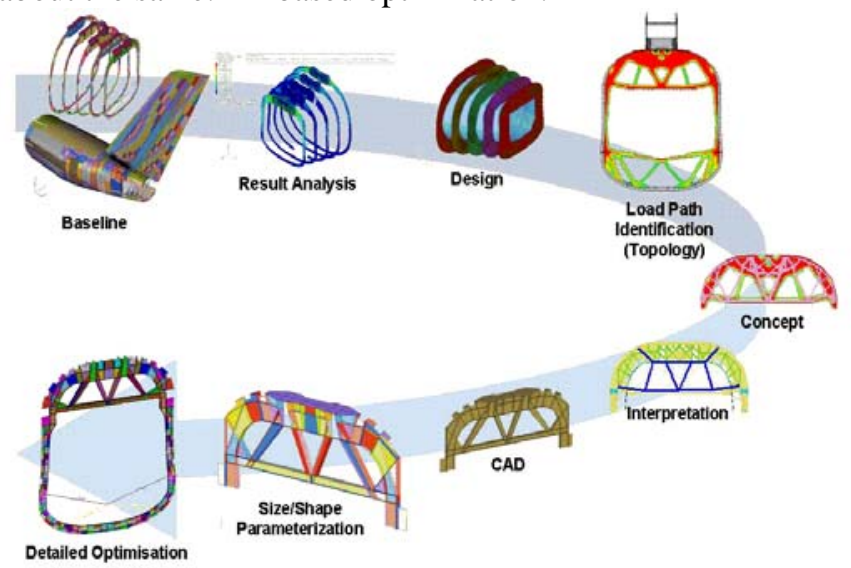

Fig. 9. Example of FE-based detail optimization when chained with topology optimization. A350XWB rear fuselage frames.

Today there is probably a lack of integration between both approaches and it would be welcome to have a joint optimization using numerical FE simulation with semianalytical stress tools. Another lack is with respect to a wide application of optimization at all skill tool levels for all structural elements. A wide stress analysis framework has 
been recently developed by AIRBUS, this framework called ISAMI (Fig. 10) is capable to address stress analysis of an entire aircraft structure. This is the proper place where local optimization should be embedded for repetitive parts, which does not exclude finite element approaches.

\section{Preliminary sizing}

For the special M5 milestone a good preliminary sizing must be ready at full component level. For this reason we need to have a good control of internal loads while using the appropriate skill tools for stress criteria. Hence the full global optimization problem must be addressed:

$$
\begin{gathered}
\operatorname{Min} \sum_{i=1}^{n_{s}} W_{i}\left(y_{i}\right) \\
\text { s.t. }\left\{\begin{array}{l}
y_{i}=f_{i}\left(x_{i}\right) \in \mathfrak{R}^{n_{i}} \\
R F_{j}\left(x_{i}, N_{k}^{i}(y)\right) \geq 1 \quad \mathrm{j}=1, \mathrm{n}_{\mathrm{c}} ; \mathrm{k}=1, \mathrm{n}_{\mathrm{lc}} \\
d_{l}(x) \geq 0 \quad \mathrm{l}=1, \mathrm{n}_{\mathrm{d}} \\
g_{m}(y) \geq 0 \quad m=1, n_{g}
\end{array}\right.
\end{gathered}
$$

Internal loads are updated and optimized for weight minimization. Global constraints (stiffness or design) can be considered. A full structure can be optimized.

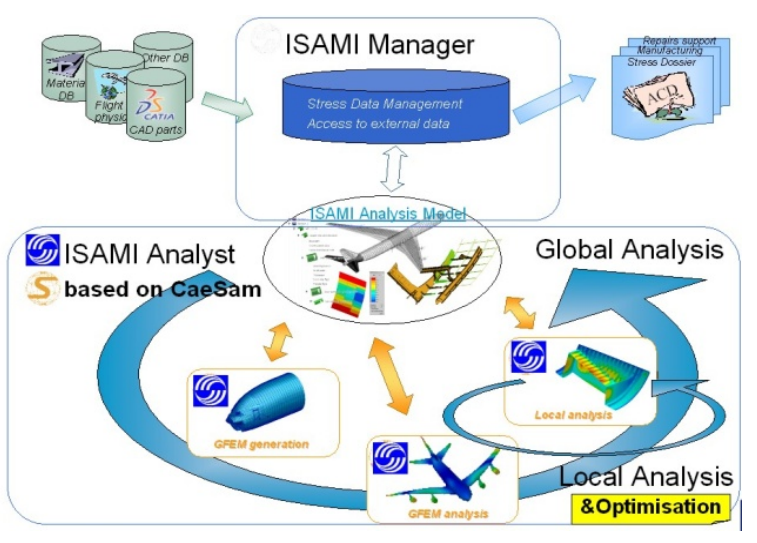

Fig. 10. ISAMI and optimization

This is the reason why AIRBUS has launched with A350 a new generation of optimization tools in order to do so. This kind of tool is integrating GFEM and skill tool analysis and makes a global/local optimization [18-22] link (Fig. 11). It was first developed for a composite wing then extended to all aircraft boxes, hence its name: COMBOX for COMposite BOXes. This tool has recently been extended to COMposite FUSElage with COMFUSE. The optimization process for COMBOX and COMFUSE is identical and is based on chain-ruling of sensitivities between global responses (internal loads) and local responses (reserve factors).

The pre-processing of an optimization process for covers was also automated. From groups of finite elements provided by the user, structural elements are built, then calculation points are assembled as groups of structural elements. Groups of structural elements are also built to define optimization regions with uniform sizing and reduce the optimization problem. To each structural element and calculation point can be attached every necessary stress information to manage the optimization and the stress analysis (materials, stringer profiles, sizing properties, calculation hypothesis).

+ Integration of GFEM and skill tools at sensitivity level

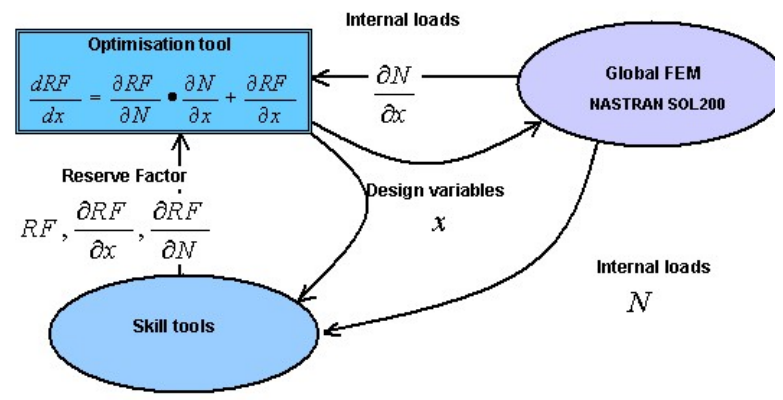

* Full sensitivity chaining to account for internal load redistribution

Fig. 11. Chain ruling of sensitivities inside COMBOX for a global-local optimization link

The pre and post-processing and the management of the stress model is addressed with a specific tool called CAESAM developed by the SAMTECH company, while the management of the full optimization process embedding NASTRAN SOL200 sensitivities and skill tools, is performed with BOSS Quattro. The optimization algorithm used is taken in the BOSS Quattro library (GCM: Globally Convergent Method). All the standard variables for composite materials are considered (Fig. 12). The total thickness and percentages for $0 \%-45^{\circ} / 45^{\circ} / 90^{\circ}$ orientations can be taken as design variables. The order of magnitude of thickness is large enough to consider a material homogenization and address the stacking sequence in a next step more manufacturing-oriented. All composite design criteria are addressed:

- buckling/post-buckling

- reparability

- damage tolerance

Buckling/post-buckling are classical criteria. They are calculated via a Rayleigh-Ritz approach, which is a precursor of the finite element method. There is an eigenvalue solver behind to estimate the global buckling of the cover elementary pattern (super-stringer). For this reason buckling analysis is the most computationally heavy part of the stress process. Reparability and damage tolerance are less usual. Reparability anticipates the possibility of small shocks on covers, which can be repaired via metallic bolted plates: reparability criteria are in fact special cases of bearing/bypass criteria for assemblies. Damage tolerance criterion considers the residual strength of composite covers after an impact specified by certification authorities and occurrence probabilities linked to the location in the aircraft and to the loading.

At this point the computational times needed for Reserve Factor sensitivity analysis is to be stressed. Indeed if we consider that this sensitivity analysis is performed via finite differences and that it is to be done for each calculation point and for each load case, the computational times can be very heavy (Fig. 13). The extension of COMBOX, called COMFUSE to fuselage is today under validation. The intent to run it on full fuselage is strong but the computational time and resources needed is today a brake. The skill tool used 
inside is more computational heavy than for boxes because it has to address the curvature of fuselage panels. Moreover the complexity of a fuselage structure is higher: there are more bays than on a wing. For this reason the application to a full fuselage will probably require hundreds of processors (Fig. 14). A decomposition approach will probably be necessary before addressing such an integrated optimization.

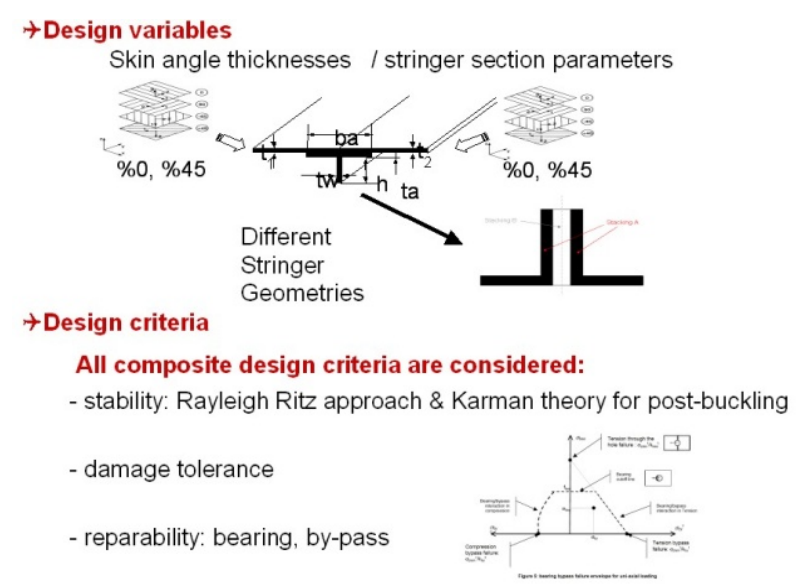

Fig. 12. Composite design \& stress process inside COMBOX $\rightarrow$ The bill for integration

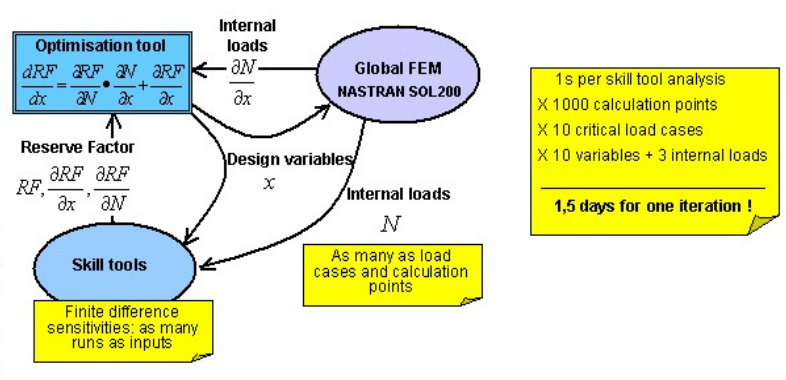

$\rightarrow$ comBOX is a powerful but computationally heavy tool

Fig. 13. Illustration of COMBOX computational times

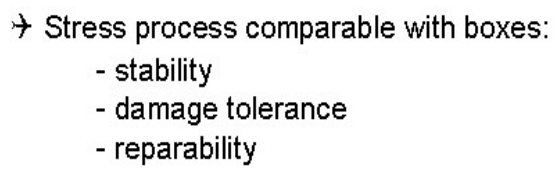

$\rightarrow$ Fuselage specificities like hat stringer profile and large damage capability taken into account

$\rightarrow$ First demonstration on centre fuselage
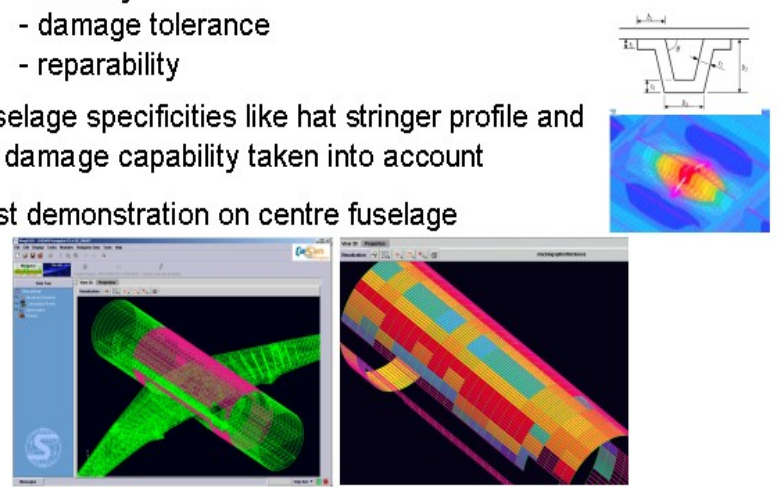

Fig. 14. Illustration of COMFUSE tool

Clearly this optimization stage appear as necessary to address the preliminary sizing at component level and be sure that the stiffness of the component and the stress are adequate for the next sizing stage where the sizing of covers will be refined or where the sizing will be extended to internal components. But this tool is clearly not what we can use today to address full aircraft sizing in multidisciplinary trade-offs. COMBOX as developed for the A350 is not the convenient tool for a rapid sizing. Meanwhile the COMBOX process itself based on a global-local optimization is to be kept in order to master both stiffness and stress in early design stages and ensure a continuity with the preliminary sizing stage.

\section{Rapid sizing}

Rapid sizing was developed at AIRBUS with such a spirit: keeping but lightening the COMBOX optimization process. In a way the topic was to reduce the COMBOX optimization process in terms of design variables and in terms of stress analysis. In another way it was to be extended to be able to address the full airframe structure with potential novel concepts.

To reduce both the design variables and stress analysis a principle of "design curves" was put in place. This principle is approximated (Fig. 15) and will be discussed further but quite efficient for this level of sizing. It consists in prior intensive local optimizations in order to derivate the minimum sizing required for a given structural pattern (here a super-stringer). Here is the principle

The minimum skin thickness and stringer area as a function of internal loads as given by the approximation is then integrated as a lower bound in the global optimization process. In this way we have built a fast sizing criteria addressing only finite element properties and not detailed stringer profiles as in the previous schemes. So the process is reduced by itself. However this approach is sometimes a rough approximation, as shown in Fig. 16. This is particularly true when thickness is driven by another criterion. For this it is possible to build design curves function of thickness. Another approach to reduce a stress criteria is to use a profile library.

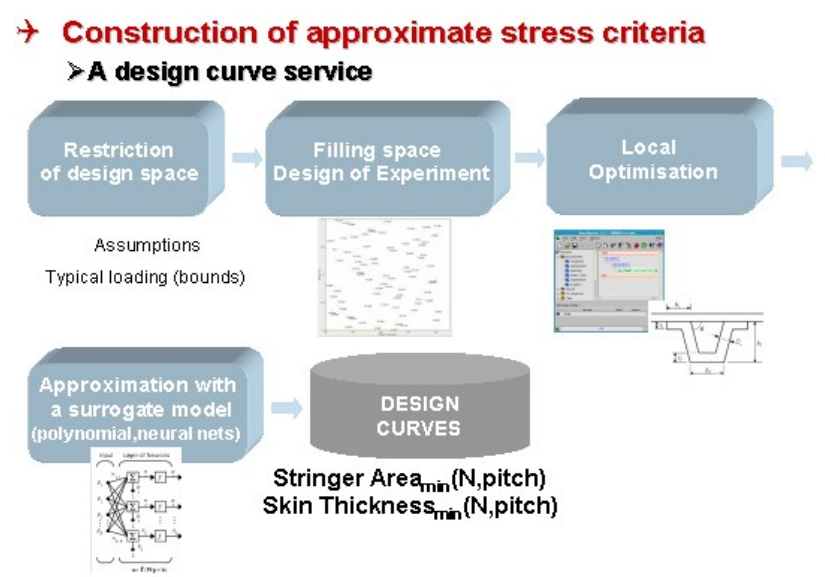

Fig. 15. Design curve mechanism 


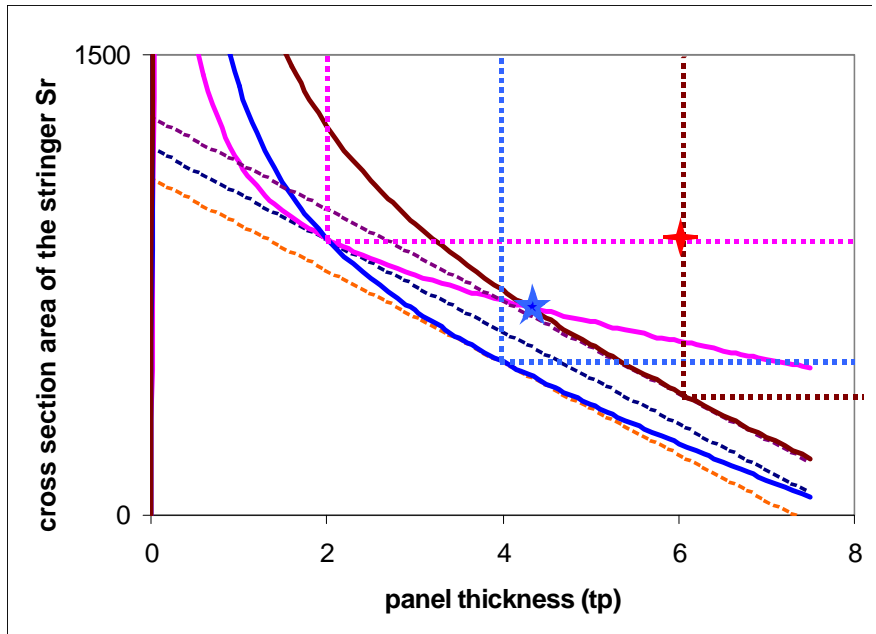

Fig. 16. Approximation of feasibility domain yielded by design curve

This rapid sizing solution was also improved in terms of scope. With COMBOX and COMFUSE today we can only address covers. The ambition with RHAPSODY (Fig. 17-18) is to address the full structure: both internal and external structure. For this reason, new Structural Elements have been added to extend the optimization process to the full structure. The full list of Structural Elements based on today structural design principles was addressed. Another important point is the flexibility in terms of stress criteria. For this we have introduced a new concept of generic skill tool, which is able to address any of the structural elements mentioned before. First example applications have been run to demonstrate the capability to address:

- different components like wing and fuselage separately

- different components simultaneously

- $\quad$ external and internal structure

\section{+ Rapid Sizing for a mixed fuselage/wing structure (A350XWB)}

Centre fuselage, centre wing box $\&$ inner wing box covers
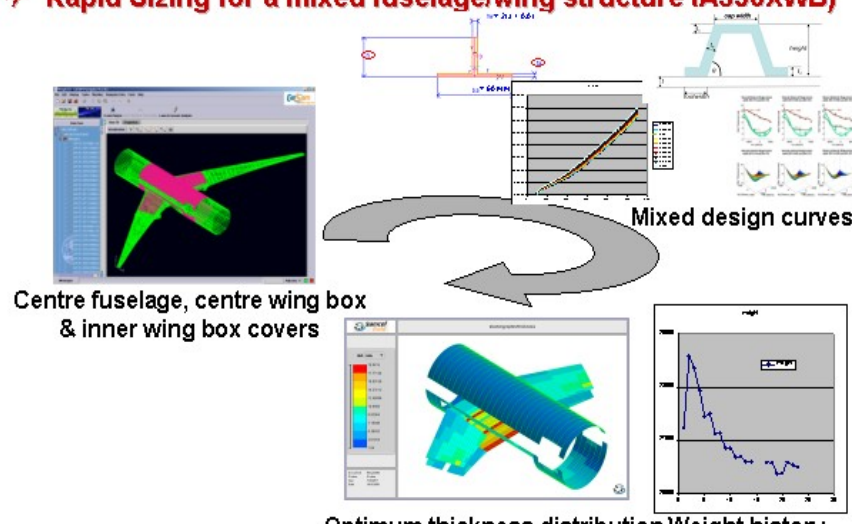

Fig. 17. Example of application with RHAPSODY (A simultaneous optimization of wing and fuselage structures was performed successfully with different design curves)

For commonality between different sizing levels inside the same interface we have kept the COMBOX framework for integration.

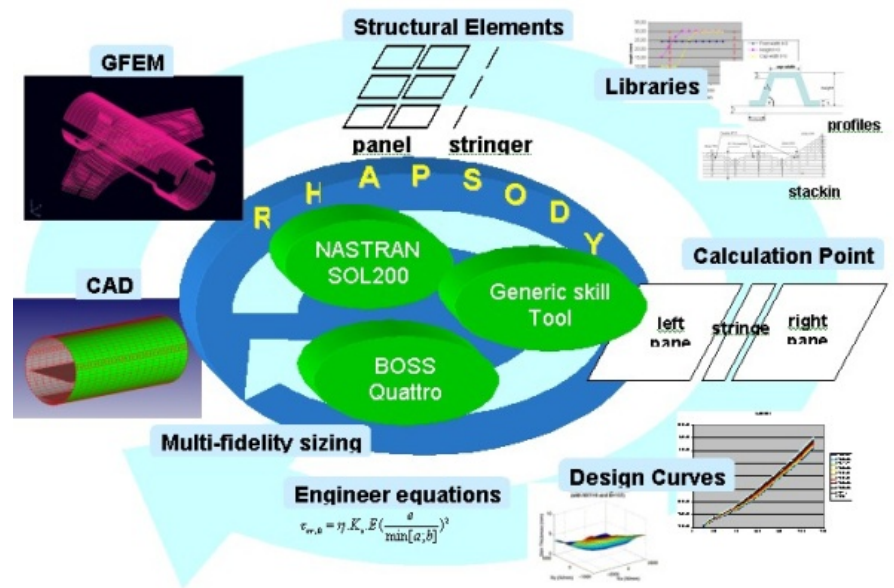

Fig. 18: RHAPSODY at a glance (With the same principles as COMBOX a lightened version was implemented focussed on Global Finite Element Model sizing)

The intent in a very next future at AIRBUS is to couple the rapid sizing capability with parameterised CAD digital mock-ups. In a further step topology optimization applied to architecture could be the source for the first CAD mock-up to have a support to architectural innovation in the numerical design process.

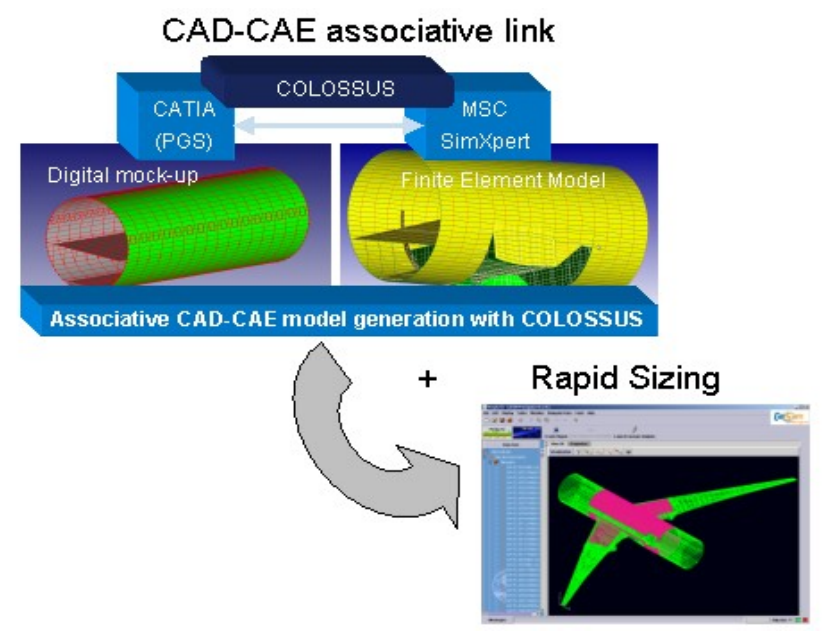

Fig. 19. A fully parameterized sizing tool

$\rightarrow$ An integrated numerical process to support architect decisions

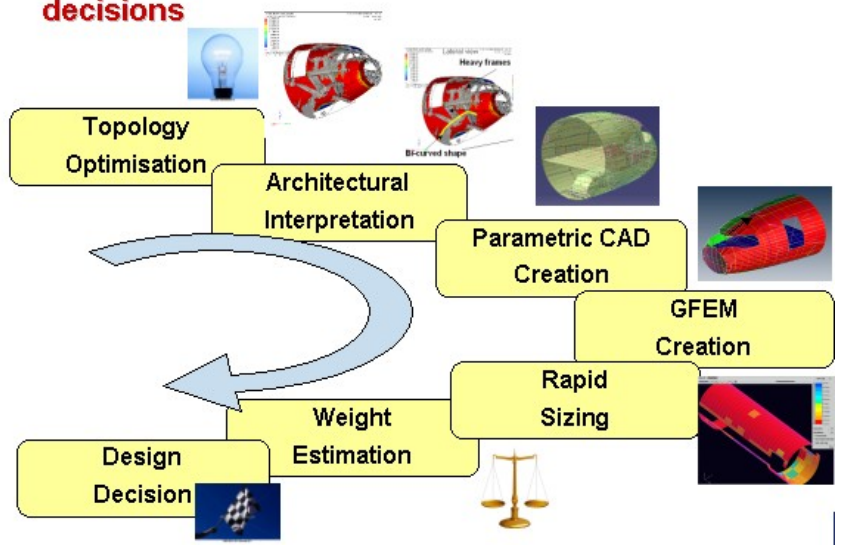

Fig. 20. From architecture creation to sizing and weight estimate 


\section{How to address composite materials}

Previous sections has described the sizing of composite structures, but has so far avoided any detailed optimization of laminates in terms of ply definitions and stacking sequences. Rather simple thickness and percentage formulations for composites were adopted along with assumptions for homogeneous and symmetric laminates. Adopting such simplistic ideas it is possible to deal with optimization of large aerospace structures using continuous methods for gradient-based optimization. However simplified approaches do not allow optimizing laminate bending stiffness properties and also do not allow us to properly account design and manufacturing rules, like stacking sequence rules and ply continuity rules. Optimized designs achieved by continuous and homogeneous approaches are therefore often considered as structural designs only and is the starting point for further design efforts to ultimately detail laminates for manufacturing. During the further detailing of the composite solution additional weight is added when rationalizing the structural optimization solution and adding details such as for example allowed ramp rates and ply continuity / ply termination rules. Clearly it would be a beneficial both in terms of both weight saving and time saving if such requirements could be dealt with by the structural optimization process.

But developing a solution for detailed optimization of composites is quite a challenge. Not only do we aim to deal with optimization of large aerospace components, like entire wing or fuselage panels, but simultaneously methods must allow a detailed definition of composite laminates in terms of both ply-definition and stacking sequences (Fig. 21-22). To complicate matters ultimately we would like to be able to utilize composite design freedom and tailor both directional properties and bending stiffness properties to the varying stress state throughout an entire structure. The problem is large scale, as we need to deal with optimization of large composite structure including optimization of cross sectional dimensions and laminate composition and ply orientations. At the same time the problem is global due to ply continuity requirements and ramp rate rules. And finally the problem is discrete due to the need to detail discrete stacking sequences. In an attempt to develop a complete process this is currently an active area of research with multiple ideas being pursued. The following presents two such ideas, but without revealing full details as these are still active areas of research.

In a first approach to deal with detailed optimization of composites it is being attempted to develop a fully continuous formulation, by using relaxation ideas from topology optimization. The basic idea is quite simple. In order to optimize a stacking sequence by continuous methods of optimization we split each ply into sub-plies, each having different orientation, and optimize the thickness of sub-plies using a penalty formulation to ensure that in the end of the optimization only a single one of the sub-plies will exist. Such approaches have with some success been already investigated in Refs (7-8). The drawback of such formulation is that they introduce a very large number of design variables. To achieve a manageable problem size for full scale aerospace applications, ideas from Refs (8-9) are being combined with the use of stacking sequence tables and to introduce additional design freedom we introduce local ply orientations as design variables. Combining multiple ideas it is possible to develop a fully continuous composite optimization formulation applicable to detailed preliminary design of large-scale composite structures keeping design variable counts reasonably to a few 1000's.

To make such ideas work for practical aerospace applications the overall calculation process needs to be developed with extensive use of intermediate variables for sensitivity analysis in order to reduce calculation requirements. The basic ideas behind this continuous optimization formulation for composite sizing and stacking sequence optimization is illustrated in Fig. 21.

In a second approach to deal with the detailed optimization of composites a two-step approach is being developed. In the first step COMBOX and/or COMFUSE are being used to develop an optimized design in terms of cross section dimensions and laminate ply percentages. In the second step pre-sizing results generated by COMBOX and/or COMFUSE are first interpreted into global ply definitions using level set ideas and a stacking sequence optimization is then being performed by simply shuffling a fixed and limited set of global ply definitions. As a key for this approach to work the COMBOX/COMFUSE pre-sizing is being performed imposing smoothness constraints, thickness ramp rate constraint and laminate percentage evolution constraints in order to ensure that ply-definitions in the second level of optimization process are continuous and well connected. Fig. 22 below illustrates how a global optimization solution via level curves are interpreted into ply definitions for a global stacking sequence optimization. This two level approach to composite optimization is still subject to further developments allowing for optimization of ply-count rounding.

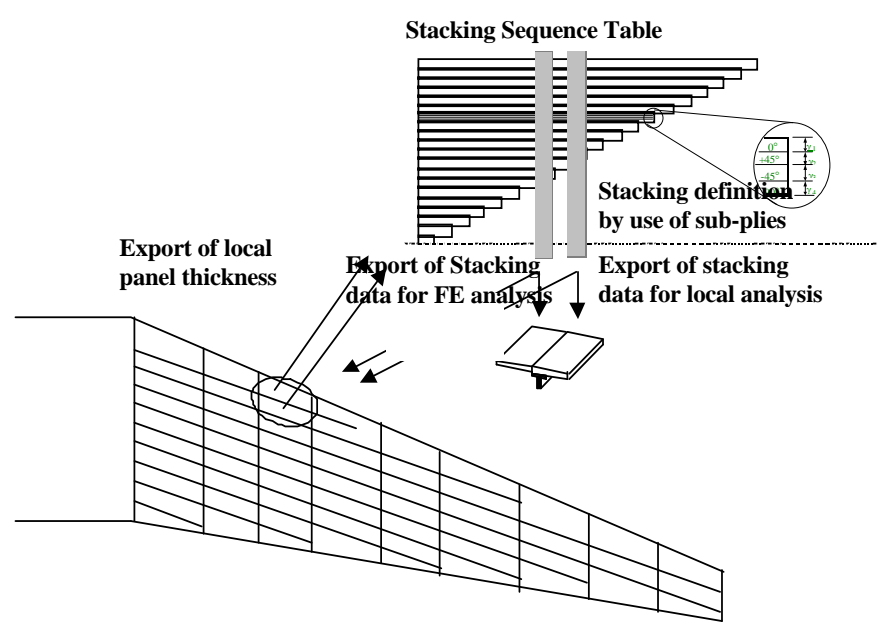

Fig. 21: Continuous approach to composite optimization 

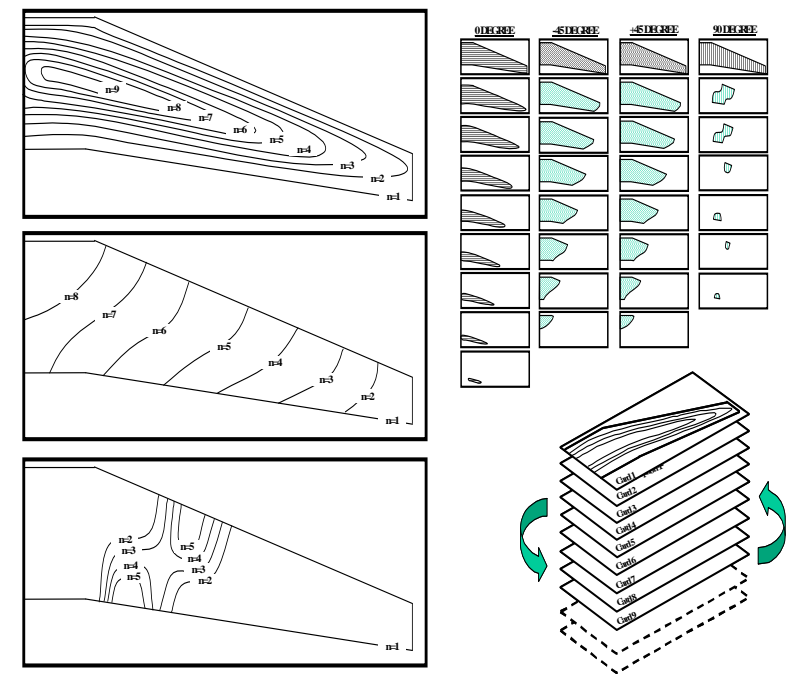

Fig. 22: Left) Thickness distributions for a $0,+/-45$ and 90 degree plies with level curves showing where $1,2,3,4$.. plies are required. Top right) Level curves are interpreted into global ply definitions. Bottom Right) A global stacking sequence optimization is performed shuffling global ply definition cards. This step is performed using a perturbation GA.

\section{How to reach a full airframe sizing}

\subsection{Decomposition/coordination (MAAXIMUS) [23]}

Whatever the algorithm used, the resolution of a continuous optimization problem via a gradient-based approach is equivalent to the resolution of the Karush-Kuhn Tucker equations which is a $\mathrm{O}\left((\mathrm{N}+\mathrm{M})^{3}\right)$ operation, with $\mathrm{N}$ the number of variables and $\mathrm{M}$ the number of constraints.

In this point of view the unique way for very large size problems to face this, is to divide $\mathrm{N}$ and $\mathrm{M}$, that is to say, to decompose the structure optimization problem in the optimization of smaller parts.

This is practically, what is done by stress people, when they size the structure structural element per structural element, except that they have no way to guarantee optimum load path or manufacturing constraints between structural elements. This is the reason why a more rigorous scheme is needed to guarantee the equivalence between the resolution of all local optimization problems and the resolution of the global one. For this we need an appropriate coordination scheme to ensure that the optimization stays correct even if decomposed.

To do so many approaches have been investigated in the literature, particularly in the area of Multidisciplinary Design Optimization. Multidisciplinary Design Optimization often uses such decomposition schemes based on disciplines (Fig. 23). One reason is that the disciplines usually have their own optimization processes, so the preferred approach is more to have a coordination of these single discipline optimizations than to solve the all-in-one problem. It is also more in adequacy with the organizational schemes of a company. $\rightarrow$ Coupling $S S O(A)^{(1)}$ processes with multi-level approaches

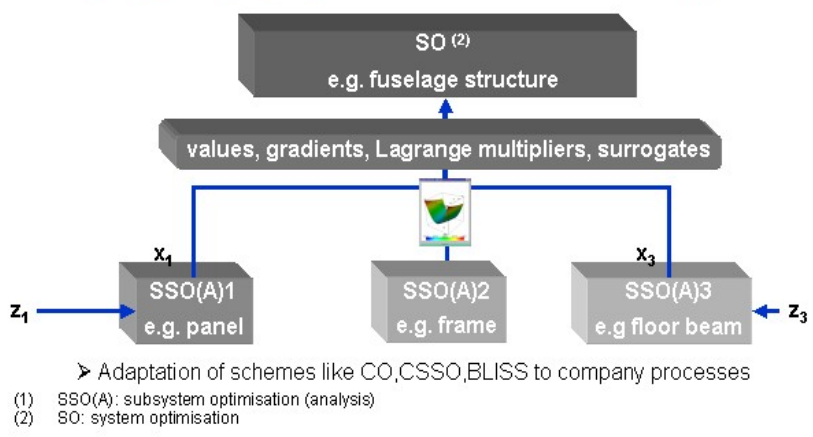

Fig. 23. Multi-level optimization applied to optimization of systems of systems for a large structure

Some preliminary successful tests were made in a previous $\mathrm{PhD}$ Thesis showing the possibility to put in place such schemes. A scheme called min-masse was put in place and has shown good results considering also heavily hyper-static configurations. Efforts are now made to consolidate this approach and extend to composite materials. One important point for such decomposition approaches is the robustness (Fig. 24). When using an all-in-one approach the difficulty is that the full structural responses and sensitivities are necessary to move to the next iteration. In a decompositioncoordination approach as the optimization is decomposed, it is easier to face an error in one of the sub-system optimizations.

Another approach is to keep the all-in-one scheme but to use as much as possible the topology of the problem (sparsity of the Jacobian matrices) in order to be able to parallelize. Approximate resolution of the Karush Kuhn-Tucker equations can also be used to limit the number of operations.

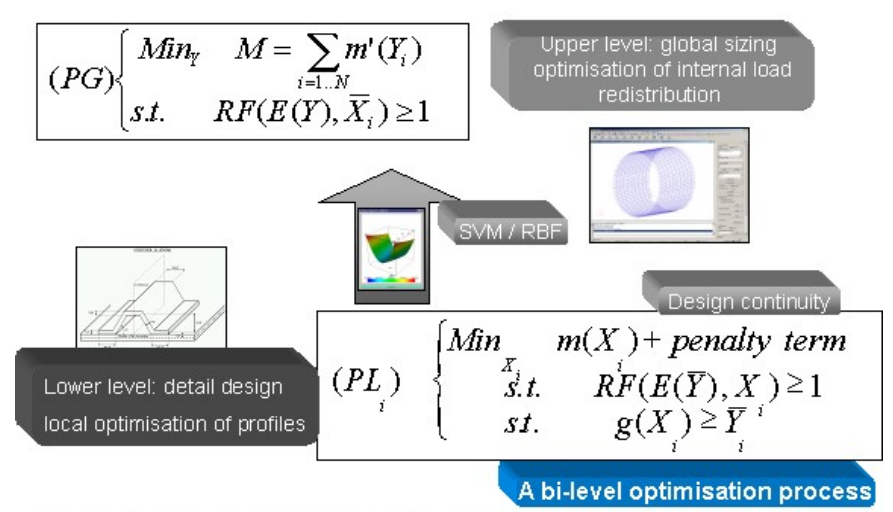

† Optimum internal load redistribution

† Natural massive parallelism decomposition

Fig. 24. A candidate solution for a multi-level decomposition scheme

\subsection{Very large size optimization approaches with parallelism (MDOW)}

A current research task within a larger program on Multi Disciplinary Optimization for Wings (MDOW) considers development of an optimization system for multi objective optimization of advanced composite structures. This task 
links the ambition to both perform very large-scale optimization and more detailed optimization of the composite structure. As part of this task a modular research framework is being developed allowing us to also experiment to with distributed computing at different levels of the optimization process and for example allow us to experiment with simple ideas for optimization decomposition.

The basic idea behind this development is to develop a number of highly efficient analysis and sensitivity analysis engines. Specific analysis modules are currently being developed for weight calculations, structural/aero-elastic analysis and for evaluation of manufacturing constraints. Each module will where required be developed to support distribute computing, in order to enable efficient management of overall runtime of the system. Fig. 25 illustrates the system set up in its most basic form, with each module drawing on a common database detailing for example the global design description and its link to an underlying FE model.

Each analysis module in Fig. 25 will perform a specific analysis and sensitivity analysis according to instructions provided in the structural optimization data base and export analysis results and sensitivity analysis results. Analysis and sensitivity analysis results are assembled to a global optimization problem description that is then passed to a gradient-based optimizer.

The modular architecture of this system, which is being created as a research framework opens up to multiple uses, with analysis/sensitivity analysis modules being used as building blocks to build/solve an overall larger optimization task. For example one could relatively easily rearrange the analysis modules such as to create an analysis process for optimization of multiple models via separate optimization streams. Also already now it would be perfectly feasible to call the structural analysis module twice in the same optimization cycle. This capability allows us to distribute computing from a very high level by managing analysis / sensitivity analysis for different load cases via individual computing streams. Also this capability allows us to call the structural analysis module with different FE models as input variables, allowing us directly to analyses and calculate sensitivities for an aircraft in different configurations.

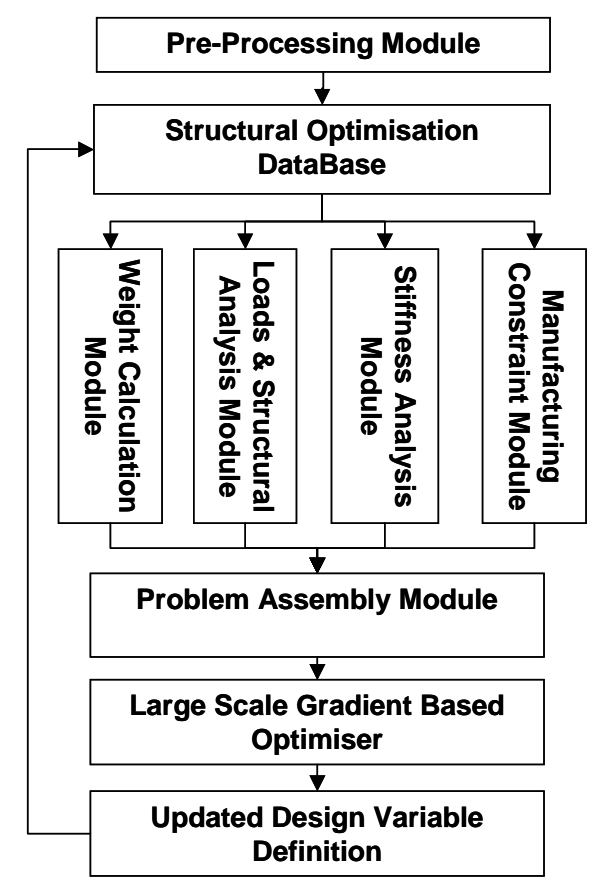

Fig. 25: Top level modular architecture and distributed computing model for optimization developments in MDOW.

A main objective of developments in MDOW is however to develop an optimization system for advanced composite optimization, allowing us to research optimization formulations such as in Section 7 before exporting ideas into industrial tools like COMBOX/COMFUSE.

\section{How to ensure a smooth transition between sizing stages}

A multi-stage sizing process for structures was presented but a few words were said in terms of continuity between the various stages. How to ensure continuity between rapid sizing and preliminary sizing, preliminary sizing and detailed sizing and last but not least detailed sizing and manufacturing. An effort was made to close the gap between rapid sizing and preliminary sizing defining specific methods for construction of rapid sizing criteria based on agreed skill tools. Two methods have been proposed for that: either a design curve construction based on approximation of reserve factors or a direct approximation of reserve factors based on the use of libraries which can be previously built. In both cases even if the agreed skill tools are used to build the simplified stress criteria there are some simplifications made for the feasible domain that are not really quantified. In the case of design curves we are not sure that the solution is really feasible at the end. In the case of profile library and reserve factor optimization, we are sure to have a feasible solution but it is not optimum because variable are linked. So an experience is to be gained in both cases to quantify the gap with preliminary sizing. This can only be done via practice. Considering the gap between the preliminary sizing and the detailed sizing, there is again an effort in terms of stress tools. It depends in fact the kind of stress tools that are used at detailed sizing level. More and more refined finite element analysis is not only used to check the structure behavior (virtual testing) but also to size this structure. In this 
point of view, it is important to understand the gap there can be between an analytical stress tool and a refined finite element modeling approach. This is to be formalized in our design and stress processes. An important point to be addressed at detailed sizing level is also the gap there can be in terms of internal load redistribution and optimum load path. Indeed detailed sizing is often made with fixed internal loads and the internal loads are thereafter updated. But this can lead to surprises showing that the separate optimums do not lead to an overall optimum. For this the schemes proposed to enable very large size optimization via decomposition-coordination have to be considered to help to control the work-shared detailed sizing. Decomposition/coordination approach must converge to a softer scheme to control a work-shared design at AIRBUS. A final point to be considered and is very important especially for composite materials is to guarantee a continuity between detailed sizing and the next phase which is manufacturing.

For that smoothness constraints have to be integrated in the optimization problems and not only at the detailed sizing levels but at any level from the rapid sizing level. Indeed manufacturing constraints can have a very large impact on structure weight. A typical example of manufacturing constraints with a large impact is the ramp-rate constraints for CFRP panels. These ramp-rate constraints limit the composite ply drop-offs over the distance: these ply dropoffs cannot be too quick or you will create a bad through thickness behaviour of your panel (interlaminate stresses). This is why some bounds are to be respected for these interlaminate constraints. An example where this kind of constraints can be very important for weight is the example of a lower wing cover: there are large thicknesses around man holes and these large thicknesses can have an impact on the full cover thickness distribution due to the ramp-rates. To summarize there are three kinds of continuity to be ensured in the multi-stage sizing processes: continuity of stress feasibility: ensure that a design for one step is feasible or not too far from feasibility for the next steps continuity of manufacturing feasibility: ensuring manufacturing constraints are satisfied at each level. Continuity of weight optimality: guaranteeing that we do not go too far from the previously obtained optimum.

\section{$\rightarrow$ A continuous design process to cover the whole airframe development}

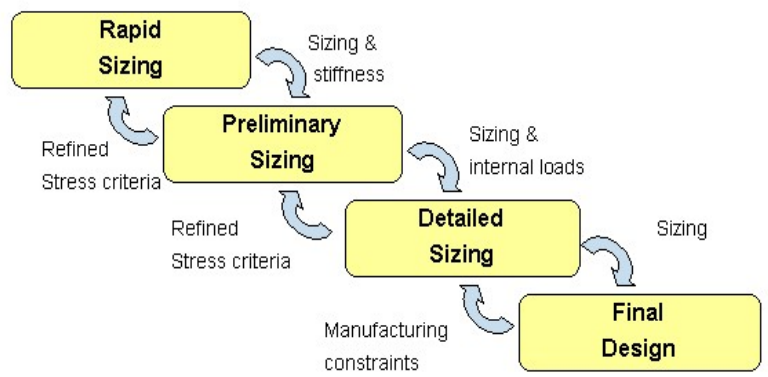

Fig. 26: Cascade of sizing stages and potential feed-backs

\section{Conclusion and ways forward}

A good set of tools for sizing has been established at AIRBUS but all challenges have not yet been addressed. A systematic process for optimization at structural element level is not yet in place and is really the starting point for a $100 \%$ integration of optimization in the design and stress process: this is the necessary building block that can be assembled to a full aircraft optimization process. This must include refined FE based analysis.

Niche applications for specific parts with 3D models will always be necessary, but there should be a way to connect stress tools. The Rapid Sizing optimization is to be pushed at full A/C level and used to prototype a full A/C preliminary sizing optimization that should go to a coordination of a work-shared detailed sizing process. Regarding composite optimization, an integration of optimization at ply level is still needed and should be put in place in the frame of MAAXIMUS project. For unconventional composite laminates, the topic is still quite open how to integrate in our processes.

\section{References}

1. L.A. Schmit, R.H. Mallet, Structural synthesis and design parameter hierarchy, Proceedings of the 3rd ASCE Conference on Electronic Computation, 269-300 (1963).

2. A. Remouchamps, Y. Radovcic, BOSS Quattro: an open system for parametric design, Structural \& Multidisciplinary Optimization, 23, 140-152 (2002).

3. B. Colson, M. Bruyneel, S. Grihon, C. Raick, A. Remouchamps, Optimization methods for advanced design of aircraft panels: a comparison, Optimization and Engineering.

4. L. Krog, A distributed computing process for optimum pre-sizing of composite aircraft box structures, Samtech 2007 User's Conference (2007).

5. AIRBUS France, MTS004. Manuel de calcul statique pour matériaux métalliques (2000).

6. A. Carpentier, S. Grihon, L. Michel, J.J. Barrau, Buckling optimization of composite panels via lay up tables, 3rd European Conference on Computational Mechanics Solids, Structures and Coupled Problems in Engineering, Lisbon (2006).

7. A. Carpentier, L. Michel, S. Grihon, J.J. Barrau, Optimization Methodology of Composite Panels. 4th European Conference on Computational Mechanics Solids, Structures and Coupled Problems in Engineering, Biarritz (2007).

8. A. Merval, M. Samuelidès, S. Grihon, Multi-level Optimization with local mass minimization, 2nd European Conference for Aerospace Sciences - EUCASS (2007).

9. A. Merval, M. Samuelidès, S. Grihon, Lagrange kuhntucker coordination for multilevel sizing of aeronautical structures, 49th conference on AIAA /ASME /ASCE /AHS /ASC Structures, Structural Dynamics \& Material Conference (2008).

10. A. Remouchamps, S. Grihon, C. Raick, B. Colson, M. Bruyneel, Numerical optimization. a design space odyssey. International Workshop, 2007: Advancements in Design Optimization of Materials, Structures and mechanical Systems, Xi'an China (2007).

11. MSC.Software, NASTRAN Linear Static Analysis User's Guide (2000).

12. SAMTECH, BOSS Quattro user's handbook - Release 
6.0-02 (2008).

13. C. Fleury, Dual methods for convex separable problems in optimization of large structural systems, Kluwer Academic Publishers, The Netherlands, 509-530 (1993)

14. M. Bruyneel, P. Duysinx, C. Fleury, A family of MMA approximations for structural optimization, Structural \& Multidisciplinary Optimization, 24, 263-276 (2002).

15. A. Merval, M. Samuelides, S. Grihon, Application of Response Surface methodology to stiffened panel optimization, 47th conference on AIAA/ASME/ASCE/AHS/ASC Structures, Structural Dynamics \& Material Conference (2006)

16. J.H. Zhu, W.H. Zhang, D. Bassir, Validity improvement of evolutionary topology optimization: procedure with element replaceable method, Int. J. Simul \& Multidisc Des Opt. 3, 347-355 (2009)

17. M. Bruyneel, A general and effective approach for the optimal design of fibres reinforced composite structures, Composites Science \& Technology, 66, 1303-1314 (2006).

18. S. Guessasma, D. Bassir, Comparing heuristic and deterministic approaches to optimise mechanical parameters of biopolymer composite materials, Mechanics of Advanced Materials and Structures. 16, 293-299 (2009).

19.S. Guessasma, D. Bassir, Optimization of mechanical properties of virtual porous solids using a hybrid approach, Acta Materiala, 58, 716-725 (2010).

20. D. Bassir, S. Guessasma, Boubakar M.L, Hybrid computational strategy based on ANN and GAPS: Application for identification of a non linear composite material, Journal of Composite Structures, 88, 262-270 (2009).

21. R.J. Balling, J. Sobieszczanski-Sobieski, An Algorithm for solving the System-level Problem in Multi-Level Optimization, NASA Contractor Report 195015 (1994).

22. L.A.Jr. Schmit, R.K. Ramanathan, Multilevel Approach to Minimum Weight Design including Buckling Constraints, AIAA Journal 16 (1978).

23. "MAAXIMUS" Integrated Project, 7th Framework, 2008-2013. 